\title{
Free electron laser with terahertz band Bragg reflectors
}

\author{
Naum S. Ginzburg, ${ }^{*}$ Andrey M. Malkin, Nikolay Yu. Peskov, Alexandr S. Sergeev, and Vladislav Yu. Zaslavsky \\ Institute of Applied Physics of RAS, Uljanov street 46, Nizhny Novgorod, 603950, Russia \\ Keichi Kamada and Ritoku Ando \\ School of Natural Science and Technology, Kanazawa University, Kanazawa, Japan
}

(Received 30 December 2008; published 3 June 2009)

\begin{abstract}
Periodical Bragg structures may be considered as an effective way of controlling the electromagnetic energy fluxes and provision of spatially coherent radiation in the free electron lasers with oversized interaction space. A new scheme of terahertz band FEL with hybrid Bragg resonator is proposed consisting of advanced input Bragg mirror and traditional output Bragg mirror. An advanced Bragg mirror exploiting the coupling between the two counterpropagating modes and the quasicutoff one provides mode selection over the transverse index. The main amplification of the wave by the electron beam takes place in the regular section of the resonator. Small reflections from the output traditional Bragg mirror are sufficient for oscillator self-excitation.
\end{abstract}

DOI: 10.1103/PhysRevSTAB.12.060702

PACS numbers: 41.60.Cr, 42.25.Fx

\section{INTRODUCTION}

Reflectors based on Bragg coupling of counterpropagating waves on the periodic structures are widely used both in quantum [1,2] and classical [3,4] electronics. In the millimeter wavelength range, the Bragg structures based on hollow metallic waveguides with periodic corrugation of inner surface allow one to combine the effective electron beam transportation with selective resonance system. However, the advance in shorter wave bands is limited because at large values of the oversize factor the coupling between numerous pairs of propagating modes occurs. As a result, the radiation generated by the electron beam would represent an uncontrolled mixture of the waveguide modes. Besides that, the absolute values of reflection coefficients decrease with the increase of oversize factors.

The problem of extending of the interaction space in the transverse direction can be partially solved by using the coupling between the propagating and the cutoff modes in the advanced Bragg reflector with additional transverse electromagnetic energy fluxes [5]. In this paper we consider the planar model of the FEL with a hybrid Bragg resonator consisting of two structures with different operating features (Fig. 1): an upstream (cathode-side) advanced Bragg reflector and a downstream (collector-side) traditional Bragg reflector. Mode control in the transverse (y) direction is achieved within the upstream advanced Bragg reflector by means of the above-described mechanism of partial waves coupling. Interaction with the electron beam oscillating in the planar wiggler mostly takes place in the regular part of the resonator, where the syn-

*ginzburg@appl.sci-nnov.ru chronous wave $A_{+}$escaping from the upstream reflector is amplified by the electron beam. A conventional 1D Bragg mirror at the output of the system reflects only a small amount of the rf energy in the backward direction, thus forming the feedback loop and providing the conditions for the self-excitation of the oscillator. According to estimations, the described above scheme can be used for realization of the powerful Bragg FEL in terahertz wave band. Unlike existing terahertz band FELs [6,7], the above scheme should be driven by a long pulse electron beam from induction linear accelerators [8] or electrostatic accelerators [9-11].

In Sec. II the properties of an advanced Bragg structure are studied and its reflection coefficient is found. In Sec. III we present the results of the simulation of nonlinear dynamics of the FEL with a hybrid Bragg resonator described above. In the Appendix some guidelines on the derivation of the basic equations are given.

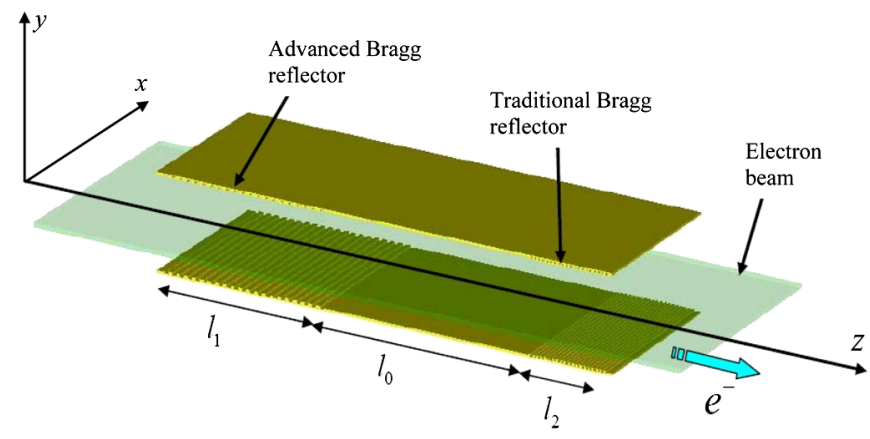

FIG. 1. (Color) Scheme of a planar FEL with hybrid Bragg resonator: 1-advanced Bragg reflector; 2-traditional Bragg reflectors; 3-electron beam. 


\section{TERAHERTZ BAND PLANAR BRAGG REFLECTORS}

An advanced Bragg reflector is formed by two parallel plates with shallow periodic corrugation of the inner walls:

$$
a(z)=a_{1} \cos \left(\bar{h}_{1} z\right)
$$

where $\bar{h}_{1}=2 \pi / d_{1}, d_{1}$ is the period of the structure (this period is 2 times larger than in traditional Bragg reflectors). Under the Bragg resonance condition

$$
\bar{h}_{1} \approx h
$$

which is satisfied when the mean distance between plates is given by $a_{0}=n d_{1} / 2$ (where $n$ is integer), the field can be presented as a sum of the two TEM modes propagating in opposite directions:

$$
\vec{E}_{ \pm}=\vec{y}_{0} \operatorname{Re}\left[A_{+}(z) e^{i(\omega t-h z)}+A_{-}(z) e^{i(\omega t+h z)}\right] .
$$

and a cutoff $\mathrm{TM}_{n}$ mode,

$$
\vec{E}=\vec{z}_{0} \operatorname{Re}\left[B(z) \sin \left(\frac{\pi n y}{a_{0}}\right) e^{i \omega t}\right]
$$

Here $\vec{y}_{0}$ is the unit vector directed normally to the surface, $\vec{z}_{0}$ is the unit vector along the propagation direction of the waves (see Fig. 1). The process of reflection via excitation of the cutoff mode can be described by the following equations (see the Appendix):

$$
\begin{gathered}
\frac{d \tilde{A}_{ \pm}}{d z} \pm i \frac{\Omega}{c} \tilde{A}_{ \pm}= \pm i \alpha_{1} \tilde{B} \\
\frac{1}{2 h} \frac{d^{2} \tilde{B}}{d z^{2}}-i \sigma \tilde{B}+\frac{\Omega}{c} \tilde{B}=\alpha_{1}\left(\tilde{A}_{+}+\tilde{A}_{-}\right) .
\end{gathered}
$$

Here $\Omega=(\omega-\bar{\omega})$ is the detuning between the Bragg frequency $\bar{\omega}=\bar{h} c$ and the frequency of the incident wave,

$$
\alpha_{1}=\frac{h a_{1}}{\sqrt{2} a_{0}}
$$

is the coupling coefficient (see Appendix for details), $l$ is the length of the structure, $\sigma=\frac{h \delta}{a_{0}}$ is the Ohmic losses parameter for the cutoff mode, $\delta$ is the skin depth (Ohmic losses for propagating waves $A_{ \pm}$are negligibly small). In Eqs. (3) and (4) the amplitudes $\tilde{A}_{ \pm}=A_{ \pm} / \sqrt{N_{A}}, \tilde{B}=$ $B / \sqrt{N_{B}}$ are normalized by the wave norms

$$
N_{A}=\frac{c a_{0}}{2 \pi}, \quad N_{B}=\frac{c a_{0}}{4 \pi} .
$$

In the case of incident wave $\tilde{A}_{+}$we apply the following boundary conditions to Eqs. (4):

$$
\begin{array}{r}
\left.\tilde{A}_{+}\right|_{z=0}=\tilde{A}_{0},\left.\quad \tilde{A}_{-}\right|_{z=l_{1}}=0 \\
{\left.\left[\frac{d \tilde{B}}{d z}-i \sqrt{2 h\left(\frac{\Omega}{c}-i \sigma\right)} \tilde{B}\right]\right|_{z=0}=0} \\
{\left.\left[\frac{d \tilde{B}}{d z}+i \sqrt{2 h\left(\frac{\Omega}{c}-i \sigma\right)} \tilde{B}\right]\right|_{z=l_{1}}=0}
\end{array}
$$

For the partial cutoff mode $\tilde{B}$, we used radiation boundary assuming that edge reflections for this wave are negligibly small. The solution of Eq. (4b) out of the Bragg structure where $\alpha_{1}=0$ is the waves propagating away from the structure: $\tilde{B} \sim e^{i \Lambda z}$ at $z<0$ and $\tilde{B} \sim e^{-i \Lambda z}$ at $z>$ 0 , where $\Lambda=\sqrt{2 h\left(\frac{\Omega}{c}-i \sigma\right)}$. The electric field is proportional to $B$ while the magnetic field is proportional to $\frac{d B}{d z}$. Taking into account the continuity of the electric and magnetic fields at the structure edges, we get the boundary condition (6b) and (6c).

Neglecting the diffraction for the cutoff mode, one can obtain the longitudinal distribution of reflected and transmitted waves from Eqs. (4) as follows:

$\tilde{A}_{-}=\tilde{A}_{0} \frac{2 i\left(\Omega^{2}-g^{2} c^{2}\right) \sin g\left(l_{1}-z\right)}{(\Omega+g c)^{2} e^{i g l_{1}}-(\Omega-g c)^{2} e^{-i g l_{1}}}$,

$\tilde{A}_{+}=\tilde{A}_{0} \frac{(\Omega+g c)^{2} e^{i g\left(l_{1}-z\right)}-(\Omega-g c)^{2} e^{-i g\left(l_{1}-z\right)}}{(\Omega+g c)^{2} e^{i g l_{1}}-(\Omega-g c)^{2} e^{-i g l_{1}}}$,

where

$$
g=\sqrt{\frac{\Omega^{2}}{c^{2}}-\frac{2 \alpha_{1}^{2} \Omega}{\Omega-i \sigma c}} .
$$

For the cutoff mode we have

$$
\tilde{B}=\frac{\alpha_{1} c\left(\tilde{A}_{+}+\tilde{A}_{-}\right)}{\Omega-i \sigma c} .
$$

The reflection coefficient is given by relation

$$
R=\frac{\tilde{A}_{-}(z=0)}{\tilde{A}_{0}}=\frac{2 i\left(\Omega^{2}-g^{2} c^{2}\right) \sin g l_{1}}{(\Omega+g c)^{2} e^{i g l_{1}}-(\Omega-g c)^{2} e^{-i g l_{1}}} .
$$

The maximum of the reflection coefficient can be written as

$$
R_{\max }=\frac{\alpha_{1}^{2} l_{1}}{\alpha_{1}^{2} l_{1}+\sigma}
$$

which corresponds to the exact Bragg resonance $(\Omega=0)$. Figure 2 shows the frequency dependencies of the reflection coefficient. Unlike the traditional Bragg structures, the decrease of the waves coupling coefficient $\alpha_{1}$ in the advanced Bragg reflector makes the reflection band narrow while the maximum of reflection coefficient does not depend on $\alpha_{1}$ at zero Ohmic losses. Thus, the coupled-wave approach shows that a narrow band reflector is effective at 


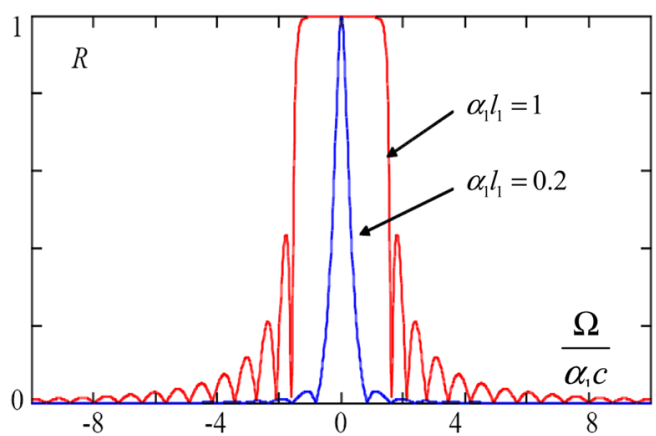

FIG. 2. (Color) Reflection coefficient vs frequency in the coupled-wave model: $\sigma=0$.

large values of oversize factor and can be realized using the coupling between propagating and cutoff modes.

The selective features of the advanced Bragg reflector are confirmed by the results of the direct simulation at terahertz frequency band with the use of 3D electromagnetic code. We took the structure period $d=0.3 \mathrm{~mm}$, distance between plates $a_{0}=6 \mathrm{~mm}$, corrugation depth $a_{1}=0.01 \mathrm{~mm}$, and reflector length $l_{1}=15 \mathrm{~mm}$. At $n=$ 40 the expected Bragg resonance frequency is $f=1 \mathrm{THz}$. In Fig. 3 the frequency dependencies of the reflection and transmission coefficients are shown for the incident TEM mode. One can see that at oversize factor $a_{0} / \lambda \approx 20$ several reflection bands corresponding to the excitation of the cutoff modes with different numbers of field variations $n$ are present. However, the reflection peak near the $1 \mathrm{THz}$ frequency corresponding to the excitation of the $\mathrm{TM}_{40}$ cutoff mode is dominant.

Note that for the effective single mode operation it is sufficient to provide a condition that the frequency distance between modes with different transverse indices $n$ exceeds the FEL amplification band

$$
c \pi / a_{0}<\omega / N
$$

which is defined by the number $N=l_{0} / d_{w}$ of wiggler periods $d_{w}$ inside the interaction space with the length $l_{0}$.

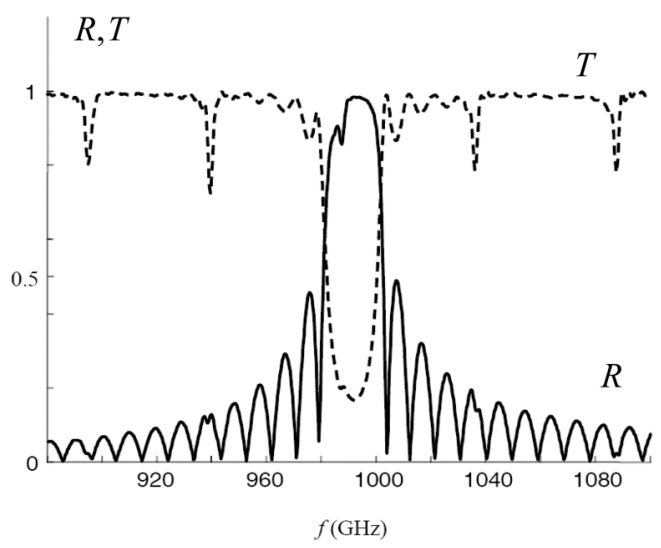

FIG. 3. Reflection (solid line) and transmission (dashed line) coefficients vs frequency in 3D simulations.
Taking into account that the FEL operation wavelength $\lambda \approx \gamma^{-2} d_{w} / 2$, we get a restriction for the width of the gap between resonator plates

$$
a_{0}<l_{0} \gamma^{-2} / 2,
$$

where $\gamma$ is relativistic mass factor. Obviously, this condition can be satisfied in terahertz wave band.

\section{NONLINEAR DYNAMICS OF THE FEL WITH AN ADVANCED BRAGG REFLECTOR}

The advanced Bragg reflector described in Sec. II should be used in the two-mirror resonator scheme as an upstream reflector (Fig. 1). To avoid large Ohmic losses associated with the excitation of cutoff mode, it is reasonable to use the conventional Bragg reflector with rather small reflectivity as a downstream reflector. Further we consider the nonlinear dynamics of the planar FEL with the hybrid resonator consisting of an upstream advanced and a downstream traditional Bragg reflector. We assume that the sheet electron beam interacts with the synchronous wave $A_{+}$.

Nonstationary equations for the amplitudes of coupled waves in the upstream reflector can be presented in the form

$$
\begin{gathered}
\frac{\partial \hat{A}_{+}}{\partial \zeta}+\frac{\partial \hat{A}_{+}}{\partial \tau}=i \hat{\alpha}_{1} \hat{B}+J \quad \frac{\partial \hat{A}_{-}}{\partial \zeta}-\frac{\partial \hat{A}_{-}}{\partial \tau}=-i \hat{\alpha}_{1} \hat{B} \\
\frac{C}{2} \frac{\partial^{2} \hat{B}}{\partial \zeta^{2}}-i \hat{\sigma} \hat{B}-i \frac{\partial \hat{B}}{\partial \tau}=\hat{\alpha}_{1}\left(\hat{A}_{+}+\hat{A}_{-}\right),
\end{gathered}
$$

where we used the following normalized variables and parameters: $\quad \zeta=C h Z, \quad \tau=t C \bar{\omega}, \quad \hat{\alpha}_{1}=\alpha_{1} / h C, \quad \hat{\sigma}=$ $\sigma / h C$

$$
\hat{A}_{ \pm}=\frac{A_{ \pm} e K \mu}{\gamma m c \omega C^{2}}, \quad \hat{B}=\frac{B e K \mu}{\gamma m c \omega C^{2}} \sqrt{\frac{N_{A}}{N_{B}}},
$$

$C=\left(\frac{e I_{0} \mu K^{2} \lambda^{2}}{8 \pi m c^{3} \gamma^{3} a_{0}}\right)^{1 / 3}$ is the gain parameter, $K=\frac{e H_{u}}{h_{w} m c^{2}}, H_{u}$ is the wiggler field amplitude, $\mu \approx \gamma^{-2}$ is the bunching parameter, $\beta_{\|}=v_{\|} / c$ is the electron translational velocity, and $\gamma$ is the relativistic mass factor. The HF current in Eq. (11) $J=\frac{1}{\pi} \int_{0}^{2 \pi} e^{-i \theta} d \theta_{0}$ can be found from the averaged electron motion equations [3]:

$$
\begin{aligned}
\left(\frac{\partial}{\partial \zeta}+\beta_{\|}^{-1} \frac{\partial}{\partial \tau}\right)^{2} \theta & =\operatorname{Re}\left(\hat{A}_{+} e^{i \theta}\right), \\
\left.\theta\right|_{\zeta=0} & =\theta_{0} \in[0,2 \pi), \\
\left.\left(\frac{\partial}{\partial \zeta}+\beta_{\|}^{-1} \frac{\partial}{\partial \tau}\right) \theta\right|_{\zeta=0} & =\Delta,
\end{aligned}
$$

where $\theta=\bar{\omega} t-h z-h_{w} z$ is the electron phase, $\Delta=$ $\left(\bar{\omega}-h v_{\|}-h_{w} v_{\|}\right) / \bar{\omega} C$ is the initial detuning of undulator synchronism, $h_{w}=\frac{2 \pi}{d_{w}}$.

For the cutoff mode we apply the radiation boundary conditions at the edges of corrugation $[12,13]$, 


$$
\begin{gathered}
{\left.\left[\hat{B}-\sqrt{\frac{C}{2 \pi i}} \int_{0}^{\tau} \frac{e^{-\hat{\sigma}\left(\tau-\tau^{\prime}\right)}}{\sqrt{\tau-\tau^{\prime}}} \frac{\partial \hat{B}\left(\tau^{\prime}\right)}{\partial \zeta} d \tau^{\prime}\right]\right|_{\zeta=0}=0,} \\
{\left.\left[\hat{B}+\sqrt{\frac{C}{2 \pi i}} \int_{0}^{\tau} \frac{e^{-\hat{\sigma}\left(\tau-\tau^{\prime}\right)}}{\sqrt{\tau-\tau^{\prime}}} \frac{\partial \hat{B}\left(\tau^{\prime}\right)}{\partial \zeta} d \tau^{\prime}\right]\right|_{\zeta=L_{1}}=0}
\end{gathered}
$$

where $L_{1}=l_{1} C h$. This condition can be obtained from (5) by means of Laplace transform [12,13].

In the output traditional Bragg reflector:

$$
a=a_{2} \cos \bar{h}_{2} z
$$

( $\bar{h}_{2}=2 \pi / d_{2}, d_{2}$ is the structure period) with the length $l_{2}$ (Fig. 1) only two counterpropagating partial waves (3a) are presented. Under the Bragg resonance condition

$$
2 h \approx \bar{h}_{2}
$$

the mutual scattering of these waves can be described as follows:

$$
\begin{array}{r}
\frac{\partial \hat{A}_{+}}{\partial \zeta}+\frac{\partial \hat{A}_{+}}{\partial \tau}-i \hat{\alpha}_{2} \hat{A}_{-}=J \\
-\frac{\partial \hat{A}_{-}}{\partial \zeta}+\frac{\partial \hat{A}_{-}}{\partial \tau}-i \hat{\alpha}_{2} \hat{A}_{+}=0,
\end{array}
$$

where $\hat{\alpha}_{2}=\frac{1}{C} \frac{a_{2}}{a_{0}}$ is the coupling coefficient. Amplification of the synchronous wave $A_{+}$in the regular section of the resonator with the length $l_{0}$ is described by the equations (12) and (16a), where one should put $\hat{\alpha}_{2}=0$.

In simulations we assert that the external energy fluxes are absent so the amplitudes of the waves $A_{ \pm}$on the corresponding boundaries turn to zero:

$$
\left.\hat{A}_{+}\right|_{\zeta=0}=0,\left.\quad \hat{A}_{-}\right|_{\zeta=L}=0,
$$

where $L=L_{1}+L_{0}+L_{2}$ is the total length of the resonator, $L_{j}=C h l_{j}$. tions:

Electron efficiency is determined by the following rela-

$$
\begin{aligned}
& \eta=\frac{C}{\mu\left(1-\gamma_{0}^{-1}\right)} \hat{\eta}, \\
& \hat{\eta}=\left.\frac{1}{2 \pi} \int_{0}^{2 \pi}\left(\frac{\partial \theta}{\partial \zeta}-\Delta\right)\right|_{\zeta=L} d \theta_{0} .
\end{aligned}
$$

In the steady-state generation regime (when $\hat{A}_{ \pm}, \hat{B} \sim$ $\left.e^{i \hat{\Omega} \tau}\right)$, one can obtain the energy conservation law from Eqs. (11)-(16) as follows:

$$
\hat{\eta}=P_{+}+P_{-}+P_{\mathrm{difr}}+\frac{\sigma}{2} \int_{0}^{L}|\hat{B}|^{2} d \zeta
$$

where

$$
P_{+}=\frac{\left|\hat{A}_{+}^{2}(\zeta=L)\right|}{4}, \quad P_{-}=\frac{\left|\hat{A}_{-}^{2}(\zeta=0)\right|}{4}
$$

are the normalized output power radiated with the partial waves $A_{+}$and $A_{-}$correspondingly,

$$
P_{\mathrm{difr}}=\frac{C}{4} \operatorname{Im}\left(\left.\hat{B}^{*} \frac{\partial \hat{B}}{\partial \zeta}\right|_{\zeta=L}-\left.\hat{B}^{*} \frac{\partial \hat{B}}{\partial \zeta}\right|_{\zeta=0}\right)
$$

is the power of diffraction losses associated with the cutoff mode $B$. Useful power is proportional to $P_{+}$, while the other terms in (17) represent various channels of energy losses.

Simulation of the FEL operation is carried out for the lengths of the input and output Bragg reflectors $l_{1}=$ $34 \mathrm{~cm}, l_{2}=17 \mathrm{~cm}$ separated by the regular section of length $l_{0}=70 \mathrm{~cm}$, for the resonator made of copper $\delta \approx$ $0.2 \mu \mathrm{m}$. For the electron energy $5 \mathrm{MeV}$ and undulator period $6 \mathrm{~cm}$, the radiation frequency is $f=1 \mathrm{THz}$. For the sheet beam current density $10 \mathrm{~A} / \mathrm{cm}$, the gap between plates $a_{0}=6 \mathrm{~mm}$ and undulator field amplitude $H_{u}=$ $5 \mathrm{kOe}$, one can obtain for the gain parameter $C \approx 1.4 \times$ $10^{-4}$. Normalized section lengths are $L_{1}=1, L_{0}=2$, $L_{2}=0.5$ and the coupling coefficients are $\hat{\alpha}_{1}=1.1, \hat{\alpha}_{2}=$ 0.8 . Results of the simulation are presented in Figs. 4-6. In Fig. 4 the process of establishment of steady-state oscillations is demonstrated. The longitudinal profile of partial waves in the steady-state regime in Fig. 5 shows that the main amplification of the synchronous wave $A_{+}$takes place after the upstream mirror. As a result, the amplitude of the cutoff mode $B$ excited in the advanced Bragg structure is relatively small. Correspondingly, Ohmic and diffraction losses associated with this mode are also small. Under such conditions, up to $95 \%$ of energy radiated by the electron beam is extracted with the running wave $A_{+}$. For the calculated efficiency of $\eta \sim 3 \%$ the power density of terahertz radiation would be $\sim 1.5 \mathrm{MW} / \mathrm{cm}$. The transient time is about $100 \mathrm{~ns}$.

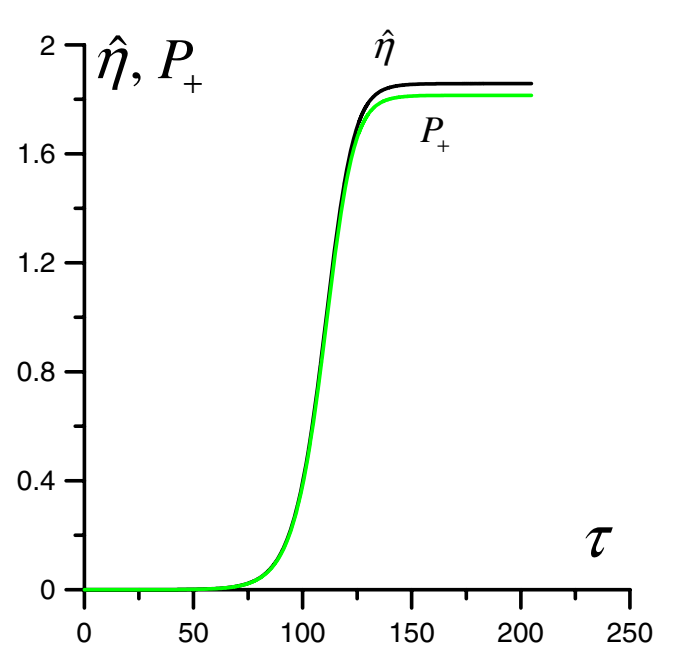

FIG. 4. (Color) Temporal dependence of efficiency (black line) and normalized output power (green line) at $L_{1}=1, L_{0}=2$, $L_{2}=0.5, \hat{\alpha}_{1}=1.1, \hat{\alpha}_{2}=0.8, \Delta=-1$, and $\hat{\sigma}=0.1$. 


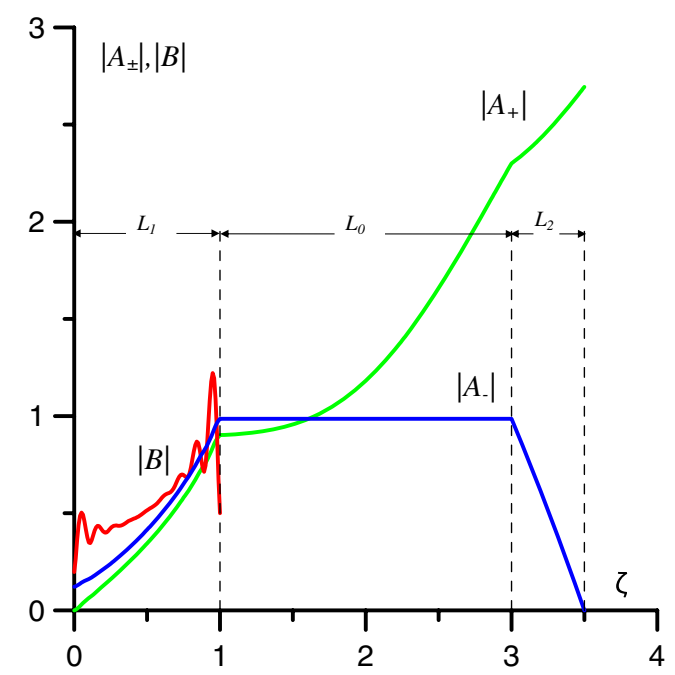

FIG. 5. (Color) Longitudinal profile of partial waves in the steady-state regime $A_{+}$(green line), $A_{-}$(blue line), and $B$ (red line) $L_{1}=1, L_{0}=2, L_{2}=0.5, \hat{\alpha}_{1}=1.1, \hat{\alpha}_{2}=0.8, \Delta=-1$, and $\hat{\sigma}=0.1$.

The simulation of the dynamics of the novel FEL scheme also shows the possibility of the effective mode control over the longitudinal coordinate. As one can see from Fig. 6, the oscillation frequency is close to the cutoff frequency of the mode $B$ and changes smoothly with the variation of detuning parameter $\Delta$ which corresponds to the variation of electron initial energy. The oscillation frequency jumps typical for a pattern with longitudinal mode competition are absent.

Note in conclusion that for the suggested scheme the upstream advanced Bragg structure provides mode control over the narrow $(y)$ transverse coordinate. The synchronization of radiation over the second transverse coordinate $x$ can be achieved due to diffraction of cutoff mode $B$ over this coordinate (to be published elsewhere).

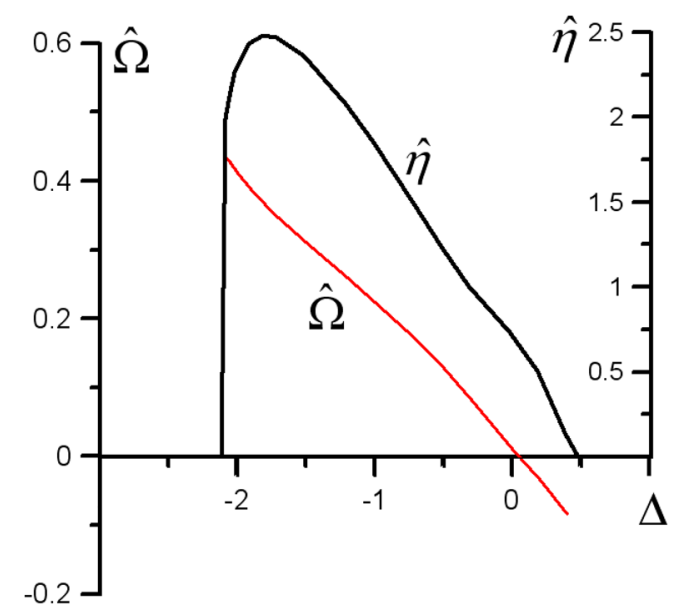

FIG. 6. (Color) Dependence of efficiency (black line) and frequency (red line) on the detuning parameter $\Delta$ at $L_{1}=1, L_{0}=$ $2, L_{2}=0.5, \hat{\alpha}_{1}=\hat{\alpha}_{2}=0.8$, and $\hat{\sigma}=0.1$.

\section{ACKNOWLEDGMENTS}

This work is partially supported by the Russian Foundation for Basic Research (Grant No. 06-02-17129) and by the Dynasty Foundation.

\section{APPENDIX}

To derive Eqs. (4) which describe coupling between the two propagating and a cutoff mode, we use the concept developed in $[14,15]$. The corrugated wall surface of the waveguide $a=a_{0}+\tilde{a}(z)$ under the assumption of shallow corrugation: $|\tilde{a}(z)| \ll \lambda, a_{0}$ can be accounted for as a noncorrugated surface with the boundary condition (which follows from the circuital theorem)

$$
\vec{E}_{\tau}=\vec{n} \times\left(\nabla\left[\tilde{a}(z) E_{n}\right]+i \frac{\omega}{c} \tilde{a}(z)(\vec{n} \times \vec{H})\right),
$$

where $\vec{n}=\vec{y}_{0}$ is the unit vector of the normal to the unperturbed (noncorrugated) waveguide wall, and $E_{n}=$ $E_{y}=(\vec{E} \vec{n})$ is the electric field component normal to the waveguide wall. This boundary condition can be substituted by the surface magnetic current,

$$
\vec{i}^{m}=\frac{c}{4 \pi} \vec{E}_{\tau}=\frac{c}{4 \pi} \vec{n} \times\left(\nabla\left[\tilde{a}(z) E_{y}\right]+i \frac{\omega}{c} \tilde{a}(z)(\vec{n} \times \vec{H})\right),
$$

distributed over the unperturbed waveguide walls.

Thus, the problem of the mode coupling in the corrugated waveguide can be reduced to the problem of mode coupling in the regular waveguide with a magnetic current (A2) induced in its walls. The equation of excitation of the electromagnetic field in the waveguide by the surface magnetic current can be presented in a form (we assume here that only one plate is corrugated)

$$
\Delta \vec{H}+\frac{\omega^{2}}{c^{2}} \vec{H}=\frac{i 4 \pi \omega \vec{i}^{m}}{c^{2}} \delta\left(y-b_{0}\right) .
$$

The solution of (A3) can be expanded into the series of transverse mode structures of the regular waveguide:

$$
\vec{H}=\sum_{j=1}^{\infty} C_{j}(z) \vec{H}_{j}^{0}(x, y) e^{i \omega t},
$$

where $\Delta_{\perp} \vec{H}_{j}^{0}+\frac{\omega_{j}^{2}}{c^{2}} \vec{H}_{j}^{0}=0, \omega_{j}$ is the cutoff frequency of the $j$ th mode. The set of eigenmodes of a regular waveguide is full and orthogonal.

If the frequency is close to the cutoff frequency of one of the modes $\omega \approx \omega_{B}$, then, after multiplying the equation (A3) by the complex conjugate structure of cutoff mode (index $B$ ), and integrating over the waveguide cross section, we obtain (compare to [15])

$$
\frac{c}{2 \omega} \frac{d^{2} B}{d z^{2}}+\frac{\omega-\omega_{B}}{c} B=\frac{1}{N_{B}} \oint \vec{i}_{\omega}^{m} \vec{H}_{B}^{0 *} d x
$$


where integration in the right-hand side over the cross section is reduced to integration over the waveguide perimeter, $N_{B}=\frac{c}{2 \pi} \int_{S_{\perp}} \vec{H}_{B}^{0} \vec{H}_{B}^{*} d S_{\perp}$ is the norm of the cutoff mode.

By the same method the excitation equation for the propagating modes (index $A$ ) can be presented as $[3,15]$

$$
\pm \frac{d A_{ \pm}}{d z}+i \frac{\omega-\omega_{c}}{c} A_{ \pm}=\frac{i \omega}{c N_{A}} \oint \vec{i}_{\omega}^{m} \vec{H}_{A}^{0 *} d x
$$

where $N_{A}=\frac{c}{2 \pi} \int_{S_{\perp}} \vec{E}_{A}^{0} \times\left.\vec{H}_{A}^{0^{*}}\right|_{z} d S_{\perp}$ is the waveguide norm.

In the assumption that the Bragg resonance conditions (2) are satisfied, the cutoff mode $B$ is coupled to the two counterpropagating modes $A_{ \pm}$, and the sum (A4) in the case of sinusoidal corrugation given by (1) can be constrained to three components:

$$
\begin{aligned}
\vec{H}= & \operatorname{Re}\left\{\left[A_{+}(z) \vec{H}_{A}^{0}(y) e^{-i h z}+A_{-}(z) \vec{H}_{A}^{0}(y) e^{i h z}\right.\right. \\
& \left.\left.+B(z) \vec{H}_{B}^{0}(y)\right] e^{i \omega t}\right\} .
\end{aligned}
$$

Substituting in Eqs. (A5) and (A6) the expression for surface current (A2) and taking into account that in planar geometry for the cutoff mode the normal $(y)$ components of electrical and magnetic fields are equal to zero, we transform excitation equations to the form

$$
\begin{aligned}
& \frac{c}{2 \omega} \frac{d^{2} B_{c}}{d z^{2}}+\frac{\omega-\omega_{c}}{c} B_{c} \\
& =\frac{\omega a_{1}}{8 \pi N_{B}} \oint\left[A_{+} \vec{H}_{A \tau}^{0} \vec{H}_{B \tau}^{0 *} e^{-i \varepsilon z}+A_{-} \vec{H}_{A \tau}^{0} \vec{H}_{B \tau}^{0 *} e^{i \varepsilon z}\right] d x \\
& \quad \pm \frac{d \hat{A}_{ \pm}}{d z}+i \frac{\omega-\omega_{c}}{c} \hat{A}_{ \pm}=\frac{i \omega a_{1}}{8 \pi N_{A}} \oint B_{c} \vec{H}_{B \tau}^{0} \vec{H}_{A \tau}^{0 *} e^{ \pm i \varepsilon z} d x .
\end{aligned}
$$

Here $\varepsilon=\left(\omega_{c}-\bar{\omega}\right) / c$ is the mismatch between the Bragg frequency and the cutoff one. After renormalizing the field amplitudes $\tilde{A}_{ \pm}=A_{ \pm} / \sqrt{N_{A}}$ and $\tilde{B}=B / \sqrt{N_{B}}$, we get

$$
\begin{aligned}
& \frac{c}{2 \omega} \frac{d^{2} \tilde{B}_{c}}{d z^{2}}+\frac{\omega-\omega_{c}}{c} \tilde{B}_{c}=\alpha\left(\tilde{A}_{+} e^{-i \varepsilon z}+\tilde{A}_{-} e^{i \varepsilon z}\right) \\
& \pm \frac{d \tilde{A}_{ \pm}}{d z}+i \frac{\omega-\omega_{c}}{c} \tilde{A}_{ \pm}=i \alpha \tilde{B} e^{ \pm i \varepsilon z}
\end{aligned}
$$

where

$$
\alpha=\frac{\omega b_{1}}{8 \pi \sqrt{N_{B} N_{A}}} \oint \vec{H}_{B \tau}^{0} \vec{H}_{A \tau}^{0 *} d x
$$

is the coupling coefficient.

For the $\mathrm{TM}_{p}$ modes $(p=0,1,2, \ldots$, including the TEM mode: $p=0$ ) mode structures are given by

$$
\vec{H}_{\tau}^{0}=\vec{x}_{0} \cos \frac{p \pi y}{a_{0}} .
$$

For the $\mathrm{TE}_{p}$ modes $(p=1,2, \ldots)$

$$
\vec{H}_{\tau}^{0}=\vec{z}_{0} \frac{p \pi c}{\omega} \cos \frac{p \pi y}{a_{0}} .
$$

Thus, for the coupling of the TM modes with indices $q$ and $p$ in the planar waveguide, the coupling coefficient is

$$
\alpha_{\mathrm{TM}_{q}-\mathrm{TM}_{p}}=\frac{k a_{1}}{2 \sqrt{1+\delta_{p 0}} a_{0}} \sqrt{\frac{k}{h}},
$$

where $\delta_{p 0}=1$ at $p=0$ and $\delta_{p 0}=0$ at $p \neq 0$,

$$
\alpha_{\mathrm{TE}_{p}-\mathrm{TE}_{q}}=\frac{p q \pi^{2}}{k} \frac{a_{1}}{2 a_{0}^{3}} \sqrt{\frac{k}{h}}
$$

for the coupling of TE modes. Note that for the coupling of TE modes the coupling coefficient is much smaller than for the coupling of TM modes and $\alpha_{\mathrm{TM}-\mathrm{TE}}=0$ for the coupling of TM and TE modes.

In the case of the two corrugated plates, the coupling coefficients (A9) and (A10) should be multiplied by the factor $F=\left(1+e^{i(\phi+\pi p+\pi q)}\right)$, where $\phi$ is the phase shift between plates' corrugations. To obtain Eqs. (4) we put $\varepsilon=0$ and consider a case of coupling between the TEM and $\mathrm{TM}_{q}$ modes in the waveguide with the two corrugated plates with cophasal corrugation for which $F=2$ for odd cutoff modes and $F=0$ for even cutoff modes.

Similar to [13], nonlinear time-domain equations (11) can be obtained by introducing the electron current into Eqs. (A8) and performing the Laplace transform to substitute frequency mismatches in the left-hand parts of the equations by the time derivatives. Boundary conditions (13) can similarly be obtained from boundary condition (6b) and (6c) by means of the Laplace transform.

[1] H. Kogelnik and C. V. Shank, J. Appl. Phys. 43, 2327 (1972).

[2] A. Yariv, Quantum Electronics (Wiley, New York, 1975).

[3] N. S. Ginzburg, V.L. Bratman, G. G. Denisov, and M. I. Petelin, IEEE J. Quantum Electron. 19, 282 (1983).

[4] N. S. Ginzburg, A. A. Kaminsky, A. K. Kaminsky, N. Yu. Peskov, S. N. Sedykh, A.P. Sergeev, and A.S. Sergeev, Phys. Rev. Lett. 84, 3574 (2000).

[5] N.S. Ginzburg, A. M. Malkin, A. A. Kaminsky, A. K. Kaminsky, N. Yu. Peskov, S. N. Sedykh, A.P. Sergeev, and A.S. Sergeev, Phys. Rev. ST Accel. Beams 8, 040705 (2005).

[6] V.P. Bolotin, N. A. Vinokurov, and D. A. Kayran, Nucl. Instrum. Methods Phys. Res., Sect. A 543, 81 (2005).

[7] Y. U. Jeong, G. M. Kazakevitch, B. C. Lee, S. H. Park, and H. J. Cha, Nucl. Instrum. Methods Phys. Res., Sect. A 528, 88 (2004).

[8] N.C. Christofilos, R.E. Hester, W. A.S. Lamb, D. D. Reagan, W. A. Sherwood, and R.E. Wright, Rev. Sci. Instrum. 35, 886 (1964). 
[9] L. R. Elias, G. Ramian, J. Hu, and A. Amir, Phys. Rev. Lett. 57, 424 (1986).

[10] Y. Socol, A. Gover, A. Eliran, M. Volshonok, Y. Pinhasi, B. Kapilevich, A. Yahalom, Y. Lurie, M. Kanter, M. Einat, and B. Litvak, Phys. Rev. ST Accel. Beams 8, 080701 (2005).

[11] A. Abramovich, M. Canter, A. Gover, J. S. Sokolowski, Y. M. Yakover, Y. Pinhasi, I. Schnitzer, and J. Shiloh, Phys. Rev. Lett. 82, 5257 (1999).

[12] N.S. Ginzburg, N. A. Zavolsky, G.S. Nusinovich, and
A. S. Sergeev, Sov. Radiophys. Quantum Electron 29, 89 (1986).

[13] N. S. Ginzburg, G. S. Nusinovich, and N. A. Zavolsky, Int. J. Electron. 61, 881 (1986).

[14] B.Z. Katzenelenbaum, Theory of Irregular Waveguides with Slowly Varying Parameters (USSR Academy of Sciences, Moscow, 1961) (in Russian).

[15] N. Kovalev, I. Orlova, and M. Petelin, Sov. Radiophys. Quantum Electron 11, 449 (1968). 